\title{
BMJ Open European Prevention of Alzheimer's Dementia Longitudinal Cohort Study (EPAD LCS): study protocol
}

\author{
Alina Solomon, ${ }^{1,2,3}$ Miia Kivipelto, ${ }^{1,2,3}$ José Luis Molinuevo, ${ }^{4}$ Brian Tom, ${ }^{5}$ \\ Craig W Ritchie, ${ }^{6}$ the EPAD Consortium
}

To cite: Solomon A, Kivipelto M, Molinuevo JL, et al. European Prevention of Alzheimer's Dementia Longitudinal Cohort Study (EPAD LCS): study protocol. BMJ Open 2018;8:e021017. doi:10.1136/ bmjopen-2017-021017

- Prepublication history for this paper is available online. To view these files, please visit the journal online (http://dx.doi. org/10.1136/bmjopen-2017021017).

Received 7 December 2017 Revised 11 July 2018 Accepted 31 October 2018

\section{Check for updates}

(C) Author(s) (or their employer(s)) 2018. Re-use permitted under CC BY-NC. No commercial re-use. See rights and permissions. Published by BMJ.

${ }^{1}$ Division of Clinical Geriatrics, NVS, Karolinska Institutet, Solna Sweden

${ }^{2}$ Theme Aging, Karolinska University Hospital, Solna, Sweden

${ }^{3}$ Neurology/Institute of Clinical Medicine, University of Eastern Finland, Kuopio, Finland ${ }^{4}$ BarcelonaBeta Brain Research Center, Fundació Pasqual Maragall, Barcelona, Spain ${ }^{5}$ MRC Biostatistics Unit, University of Cambridge, Cambridge, UK

${ }^{6}$ Centre for Dementia

Prevention, Centre for Clinical Brain Sciences, University of Edinburgh, Edinburgh, UK

Correspondence to

Dr Alina Solomon;

alina.solomon@ki.se

\section{ABSTRACT}

Introduction The European Prevention of Alzheimer's

Dementia (EPAD) project is funded initially by the

Innovative Medicines Initiative and has been established to overcome the major hurdles hampering drug development for secondary prevention of Alzheimer's dementia, by conducting the EPAD Longitudinal Cohort Study (LCS) in alignment with the Bayesian adaptive designed EPAD Proof-of-Concept (PoC) trial.

Methods and analysis EPAD LCS is an ongoing prospective, multicentre, pan-European longitudinal cohort study. Participants are recruited mainly from existing parent cohorts across Europe to form a 'probability-spectrum' population covering the entire continuum of anticipated probability for Alzheimer's dementia development. The primary objective of the EPAD LCS is to be a readiness cohort for the EPAD POC trial though a second major objective is to generate a comprehensive and large data set for disease modelling of preclinical and prodromal Alzheimer's disease. This characterisation of cognitive, biomarker and risk factor (genetic and environmental) status of research participants over time will provide the necessary well-phenotyped population for developing accurate longitudinal models for Alzheimer's disease covering the entire disease course and concurrently create a pool of highly characterised individuals for the EPAD PoC trial.

Ethics and dissemination The study has received the relevant approvals from numerous Institutional Review Boards across Europe. Findings will be disseminated to several target audiences, including the scientific community, research participants, patient community, general public, industry, regulatory authorities and policy-makers. Regular and coordinated releases of EPAD LCS data will be made available for analysis to help researchers improve their understanding of early Alzheimer's disease stages and facilitate collaborations. Trial registration number NCT02804789.

\section{INTRODUCTION}

Alzheimer's disease (AD) is the leading cause of dementia globally. ${ }^{1}$ As the population ages, the number of people with dementia will rise, and the economic burden of $\mathrm{AD}$ will increase dramatically from an already high baseline ( $€ 262$ billion in 2015). ${ }^{2}$ Clinical trials targeting populations with manifest

\section{Strengths and limitations of this study}

- Prospective, multicentre, pan-European longitudinal cohort study with a large sample size recruited mainly from existing parent cohorts.

- Well-phenotyped 'probability-spectrum' population covering the entire continuum of probability for Alzheimer dementia development.

- Disease modelling based on four dimensions including cognitive and other clinical features, biomarkers, risk factors (fixed and modifiable) and trajectories of change in these over time.

- Readiness population for a Bayesian adaptive designed proof-of-concept trial, with high-quality runin, pre-randomisation data against which the impact of various interventions will be measured.

- Limitations: alignment of the cohort with the proofof-concept trial means that this is not a traditional epidemiologically selected real-life population.

dementia have so far failed. ${ }^{3}$ There is now consensus that the genesis of $\mathrm{AD}$ predates dementia onset by over 20 years, ${ }^{4}$ presenting an opportunity for early disease course modification. The key challenge is to accurately identify individuals with a high probability of subsequent $\mathrm{AD}$ dementia development, who are suitable for trial inclusion and willing to participate in secondary prevention studies. Secondary prevention populations can have, for example, evidence of $\mathrm{AD}$ pathology through relevant biomarker abnormalities, but without a clinical diagnosis of dementia. ${ }^{5}$

Current proposals for defining an individual's probability for dementia development have focused mainly on the AD stage proximal to dementia onset and have relied on a very limited number of factors, for example, cognition and amyloid or tau biomarkers. ${ }^{6-10}$ Disease models and their phenotypic expression needed for probability estimation in earlier disease stages are currently less well defined. It is important to first develop accurate disease models for dementia onset or $\mathrm{AD}$ progression in early, asymptomatic or 


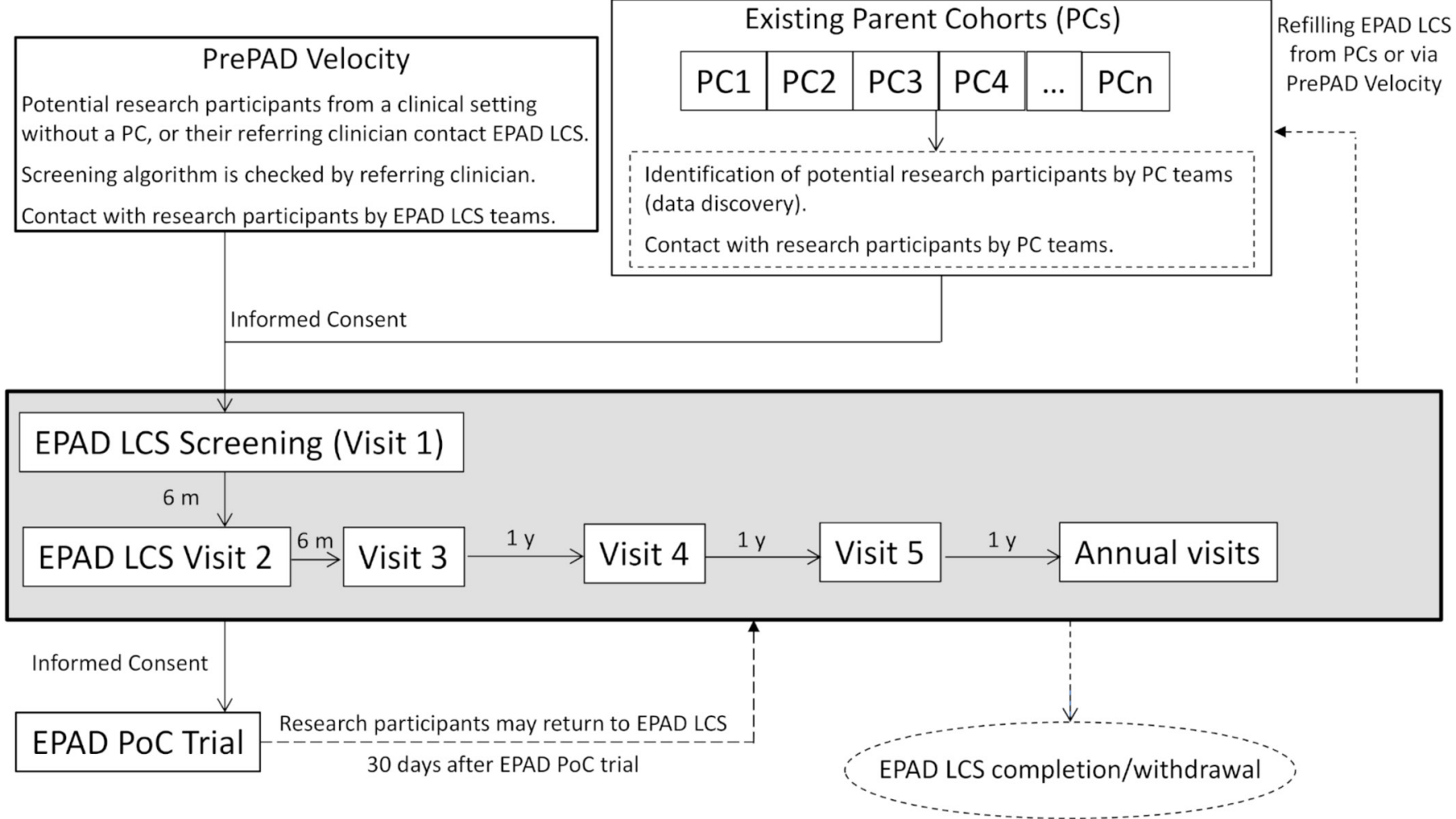

Figure 1 Flow of participants to European Prevention of Alzheimer's Dementia Longitudinal Cohort Study (EPAD LCS) and into the EPAD Proof-of-Concept (PoC) trial.

mildly symptomatic disease stages. These people need to be followed up longitudinally, after which they could be recruited into trials designed to reduce early disease burden and therein decrease the probability of developing dementia. Moreover, the refined definition of populations at risk of dementia will provide data for the optimal stratification of these populations to match onto tailored disease-modifying therapies as the basis for better personalised medicine. ${ }^{11}$

The European Prevention of Alzheimer's Dementia (EPAD) is a project to develop an environment for and then test multiple different interventions targeting the secondary prevention of $\mathrm{AD}$ dementia. ${ }^{5}$ The EPAD project is ongoing across Europe with 38 partners from academia and the commercial sector. EPAD is conducting a Longitudinal Cohort Study (EPAD LCS) in alignment with a Bayesian adaptive designed EPAD Proof-of-Concept (PoC) trial (figure 1). This article presents the EPAD LCS study protocol.

\section{OBJECTIVES OF EPAD LCS}

EPAD LCS is a prospective, multicentre, pan-European cohort study that will address the dual need to develop accurate longitudinal models for $\mathrm{AD}$ covering the entire disease course and to create a pool of highly characterised individuals for potential recruitment into the EPAD PoC trial. EPAD LCS will have a well-phenotyped "probability-spectrum' population, that is, covering the entire continuum of probability for dementia development, from low to high and everywhere in between.

EPAD LCS has four main objectives:

1. To provide a well-phenotyped population (readiness population) for the EPAD PoC trial to minimise trial screening failures.

2. To provide a well-phenotyped probability-spectrum population for developing and continuously improving disease models for $\mathrm{AD}$ in individuals without dementia. Probability for subsequent dementia will consider four different dimensions: cognitive and other clinical features, biomarkers, risk factors (fixed and modifiable) and trajectories of change in these over time.

3. To use disease models for assessing where and why participants fall in the overall probability continuum, and thereafter inform selection of participants into the EPAD PoC trial.

4. To provide high-quality run-in, pre-randomisation data for the EPAD PoC trial against which the impact of various interventions is measured.

\section{EPAD LCS STUDY DESIGN AND METHODS}

Recruitment sources for EPAD LCS

EPAD LCS participants will be recruited mainly from existing parent cohorts (PCs) across Europe. These can be research cohorts (eg, observational studies with participants from the general population or other populations, 
prevention trials, or pre-existing readiness cohorts) or clinical/routine care cohorts (memory clinic or general practitioner/primary care based). Cohort eligibility criteria are active cohorts including participants without dementia aged at least 50 years; willingness of the principal investigator of the PC to provide research participants for EPAD LCS and EPAD PoC trial; and existing consent from participants for re-contact by Parent Cohort team, or possibility to obtain consent to re-contact by PC team.

To ensure PC engagement, they will be selected based on close connections with core partners in the EPAD Consortium, maximally leveraging those involved in European Medical Information Framework (EMIF) and regional initiatives like the Dementias Platform UK (DPUK). Many other cohorts will also be included as needed.

Recruitment from existing PCs will be complemented with participants coming directly from clinical settings without a PC.

The involvement of existing PCs and clinics where some data are already available on potential participants will facilitate fast recruitment. In addition, the variety of recruitment sources (from general populations to memory clinics) will provide a probability-spectrum population covering the entire continuum of probability for $\mathrm{AD}$ dementia development.

\section{EPAD LCS study population}

EPAD LCS eligibility and exclusion criteria are listed in table 1.

EPAD LCS will be maintained over time by continuous refilling from the PCs or clinical settings as participants move into the PoC trial or drop out. Initial duration of EPAD LCS will be from May 2016 (start of recruitment) to December 2019 (end of current Innovative Medicines Initiative (IMI) funding), and after that extension of consent will be asked from participants who are still eligible for EPAD LCS. EPAD LCS participants will not be asked to leave their PCs. Participants recruited into the EPAD PoC trial may return to EPAD LCS at least 30 days after trial completion, if they wish to and if they are still eligible for EPAD LCS.

The current status of the LCS can be followed on the EPAD website (http://ep-ad.org/) where updates are continuously posted as new research participants, recruiting sites and countries join the project. There are now over 1000 participants from 21 active sites in seven countries. Recruitment status as of 29 November 2018 is shown in figure 2. Recruitment rate is expected to increase as recently opened sites reach their full capacity and new sites/countries also start recruiting.

\section{EPAD LCS participant selection process}

Selection from PCs (PrePAD)

Potential EPAD LCS research participants will be identified by each PC team based on data in their own PC. Individual-level PC data do not have to be shared with EPAD.
To ease the search process, a data discovery software tool is provided to PCs by EPAD. The Participant Register for EPAD (PrePAD) solution has been developed by EPAD study partners working with EMIF and DPUK. ${ }^{12}$ PrePAD queries will be run that provide counts of participants, without giving EPAD LCS access to individual-level data. Only the PC team will be able to identify the selected PC research participants and contact them. Those who express interest in EPAD LCS participation are then referred to the local LCS site.

As of March 2018, 10 different cohorts with a total of 17500 participants aged $>50$ years and without dementia have been included in PrePAD. ${ }^{12}$ New cohorts are continuously added.

\section{Selection from clinical settings (PrePAD Velocity)}

The participant or referring clinician will contact the local EPAD LCS site directly. The referring clinician will verify eligibility using a checklist based on assessments available in each referring clinical setting.

\section{Novel flexible approach to selection}

EPAD LCS will provide a probability-spectrum population, that is, where the entire continuum from low to high probability of subsequent dementia is represented at any time during the study. Probability of developing dementia is determined by multiple dimensions, for example, cognition, biomarkers and traditional risk factors (genetic and environmental). However, no disease model covering all these dimensions is currently available to determine where an individual is located on the probability continuum. In addition, an individual may move across the continuum over time due to changes in these dimensions.

EPAD LCS needs to ensure that at any time (1) the entire probability continuum is represented, and (2) there are enough participants potentially eligible for an adaptive designed trial, where multiple active experimental drugs may be assessed concurrently with a shared placebo arm, and interim analyses may affect participant accrual or stopping/continuing trial arms. For this purpose, a flexible approach to selection will be used (box 1). This will allow for adjustments over time as data accumulate, disease models improve and the needs of the EPAD PoC trial's intervention pipeline change.

To guarantee a well-organised selection process, EPAD LCS has a Balancing Committee (biostatisticians, data managers and LCS senior investigators) responsible for data monitoring and algorithm adaptations, and an Algorithm Running Committee responsible for algorithm documenting, and sending outputs to PCs or clinics in PrePAD Velocity. ${ }^{13}$

This centralised selection process was also set up because investigators will be blinded to results of new data collected in the EPAD LCS, namely cerebrospinal fluid (CSF) biomarkers of tau and amyloid, imaging results and apolipoprotein E (APOE) $\varepsilon 4$ allele carrier status, 
Table 1 Criteria for selection of European Prevention of Alzheimer's Dementia Longitudinal Cohort Study (EPAD LCS) participants

\begin{tabular}{|c|c|}
\hline $\begin{array}{l}\text { Eligibility } \\
\text { criteria }\end{array}$ & $\begin{array}{l}\text { Age at least } 50 \text { years } \\
\text { Completing all EPAD LCS screening/baseline assessments } \\
\text { Able to read and write and with minimum } 7 \text { years of formal education } \\
\text { Willing in principle to participate in the EPAD Proof-of-Concept trial subject to further informed consent } \\
\text { Have a study partner or can identify someone willing in principle to be a study partner* }\end{array}$ \\
\hline $\begin{array}{l}\text { Exclusion } \\
\text { criteria }\end{array}$ & 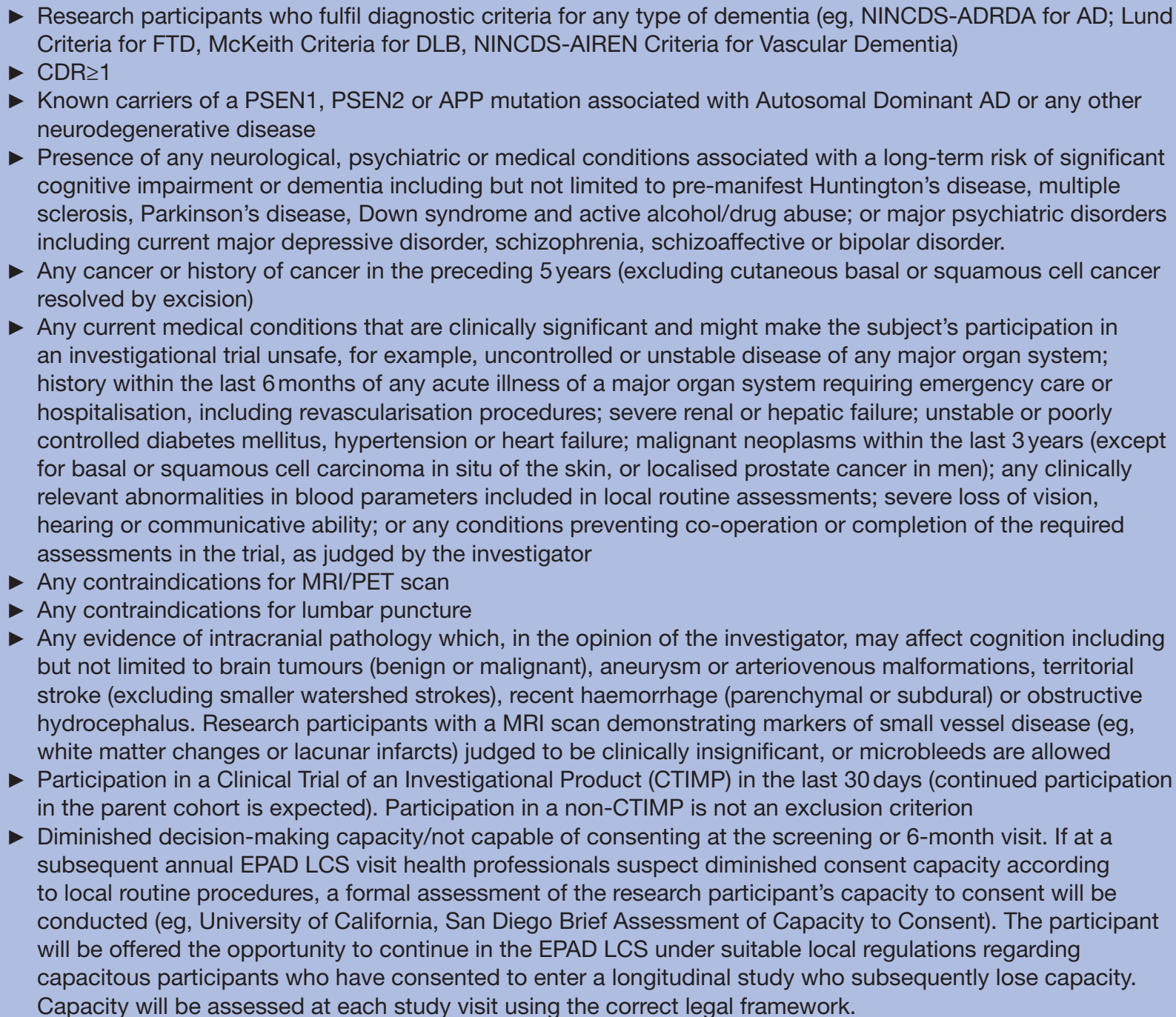 \\
\hline
\end{tabular}

${ }^{*}$ A study partner is, for example, relative or friend who is at least 18 years old, may or may not live together with the participant, and is available either for face-to-face or telephone contact with the EPAD LCS team. As EPAD LCS participants do not have dementia, have no or only slight impairment (ie, Clinical Dementia Rating, CDR 0 or 0.5 ) and are fully capable of providing informed consent (as per exclusion criteria), the primary role of the study partner in EPAD LCS will be as informant.

AD, Alzheimer's disease; APP, amyloid precursor protein; DLB, dementia with Lewy bodies; FTD, frontotemporal dementia; NINCDSADRDA, National Institute of Neurological and Communicative Disorders and Stroke-Alzheimer's Disease and Related Disorders Association (now Alzheimer's Association); NINCDS-AIREN, National Institute of Neurological Disorders and Stroke-Association Internationale pour la Recherché et l'Enseignement en Neurosciences; PSEN, presenilin.

to limit biases in clinical assessments that may affect disease modelling work in EPAD LCS. This blinding is only compromised if a research participant enters LCS via PrePAD Velocity with known and disclosed biomarker status or if the research participant enters an arm of the EPAD PoC which requires only biomarker-positive individuals.

\section{EPAD LCS outcomes and other assessments}

EPAD LCS outcomes, other assessments and the data collection schedule are detailed in tables 2 and 3 . The assessments are based on recommendations developed by the five EPAD Scientific Advisory Groups (SAGs) (Clinical and Cognitive Outcomes, Epidemiology, Fluid Biomarkers, Genetics and Imaging). SAGs 


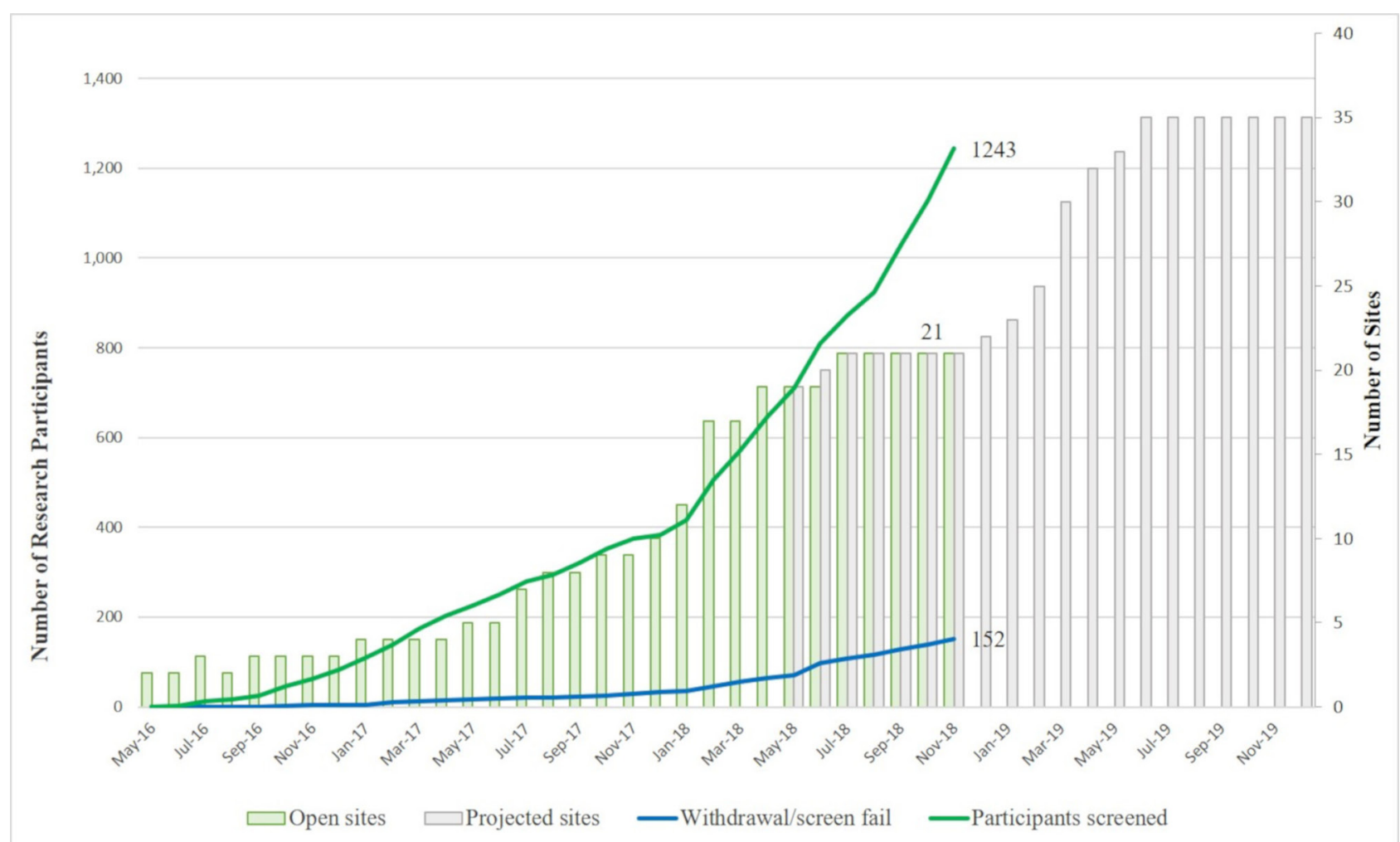

Figure 2 European Prevention of Alzheimer's Dementia Longitudinal Cohort Study (EPAD LCS) recruitment status (29 November 2018).

recommendations were based on reviewing the current literature, following widely accepted practices, and minimising participant burden.

\section{Cognitive outcomes}

The selection process for EPAD LCS cognitive outcome measures has been described previously. ${ }^{14}$ The EPAD Neuropsychological Examination (ENE) battery (table 3) was chosen to cover all relevant cognitive domains, with greatest possible sensitivity to early-stage changes. The ENE battery was also developed to be modulable, that is, to allow individual components to be selected out corresponding to specific drug targets if necessary during the EPAD PoC trial. In addition, component tasks will have four alternative forms for retesting.

For LCS purposes, primary outcomes include anchor or criterion measure(s) accepted by regulatory authorities in previous registration trials. The Repeatable Battery for the Assessment of Neuropsychological Status (RBANS) will serve as the criterion measure for this study. ${ }^{14}$ For statistical purposes, the RBANS Total Scale Index Score (table 3) will serve as the primary outcome. LCS will also help validate the secondary and exploratory cognitive outcome measures against a known and accepted criterion measure. Although the EPAD LCS is conducted in compliance with Good Clinical Practice (GCP), including the primary outcome, the computerised secondary and exploratory cognitive measures are undergoing additional validation in EPAD LCS and, thus, do not yet fully meet GCP (Title 21 CFR Part 11/European Union Annex $11)$.

\section{CSF biomarker outcomes}

Measurements will include AD-related markers (beta-amyloid, total tau and phosphorylated tau), and these data will be used for disease modelling and for staging of disease pathology. CSF sampling follows a harmonised preclinical protocol and analyses take place using the fully automatised Roche Elecsys System in a single laboratory (University of Gothenburg). Additional CSF is stored in the EPAD BioBank at the Roslin Research Institute, University of Edinburgh with all other fluid samples.

\section{Neuroimaging outcomes}

The selection process for the neuroimaging measures included in the LCS protocol was based on evidence from available studies with an emphasis on secondary prevention of $\mathrm{AD}$. Other considerations were usefulness of imaging data for the EPAD PoC trial, participant burden, implementation and costs, and avoiding redundancies between imaging and non-imaging measures.

The MRI acquisition is divided into

1. Core image acquisition, conducted in all LCS participants to assess study eligibility, for baseline assessment that can be used for subsequent safety monitoring in the EPAD PoC trial, and for quantitative analysis of brain structure and vascular lesions. Alzheimer's Disease Neuroimaging Initiative (ADNI)-like protocols and 
Box 1 Novel flexible approach to participant selection

\section{Flexible algorithm for identification of potential} participants from parent cohorts (PCs)

- For example, probability of subsequent dementia (and the selection algorithm) may be initially based on age, absence of dementia diagnosis and family history of Alzheimer's disease (AD) in a PC with less extensive assessments; or age, cognitive performance and APOE genotype in another PC with more detailed assessments; or age, cognitive performance, MRI and cerebrospinal fluid biomarkers in a $\mathrm{PC}$ where such data are available.

- The Participant Register for EPAD (PrePAD) queries of PCs will be conducted potentially every month and may be adjusted depending on several factors: types of available data in the PC; the structure of the probability spectrum at any given time point in European Prevention of Alzheimer's Dementia Longitudinal Cohort Study (EPAD LCS); the EPAD proof-of-concept (PoC) trial's intervention pipeline; the capacity at each EPAD LCS site to baseline and manage new participants.

- The flexible algorithm will be agreed on and applied by the EPAD LCS Balancing Committee, and the output will be provided to each $\mathrm{PC}$ by the Algorithm Running Committee.

Oversampling or undersampling from different types of PCs

- For example, if some PCs are more likely to provide participants with a profile suitable for a certain PoC trial arm, oversampling from such cohorts and undersampling from others may occur before and during the trial recruitment period.

\section{Flexible algorithm and oversampling/undersampling for PrePAD Velocity}

- For similar reasons, a central element of PrePAD Velocity will be that the $A D$ biomarker status of referred patients should be known from their regular clinical assessments.

- The selection algorithm will be agreed on by the Balancing Committee based on information about assessments available in each referring clinical setting. The Algorithm Running Committee will provide a checklist to the referring clinician for verifying eligibility before contacting the local EPAD LCS site.

\section{Flexible algorithm for refilling EPAD LCS over time}

- The aforementioned procedures will be applied for both establishing and refilling the EPAD LCS.

- The structure of the probability spectrum in LCS may change over time because participants (1) move into the PoC trial, (2) drop out or (3) their characteristics (eg, cognition, biomarkers, risk factors) change.

Depending on the structure of the probability spectrum at any given time point in LCS, participants coming in may or may not need to match participants moving out.

quality control will be used to ascertain precision in measuring change.

2. Advanced image acquisition, which only a subset of sites with suitable equipment and experience will acquire. This may include one or more of the following types of acquisition: 3D susceptibility weighted imaging or 3D-T2*, diffusion tensor imaging, arterial spin labelling and resting state functional MRI.

\section{Genetic assessments}

The primary genetic assessment will include APOE genotype. The samples may also be sequenced when additional resources become available. Genetic variants with strong effect (eg, APP, PSEN1 and 2) are too rare in the population to justify testing in the EPAD LCS. In addition, most of these rare mutations are observed in individuals with early-onset $\mathrm{AD}$ and are therefore unlikely to be included in the EPAD LCS.

\section{Other assessments}

A broad range of sociodemographic, medical and lifestyle-related data will be collected (tables 2 and 3). MiniMental Status Examination ${ }^{15}$ and Clinical Dementia Rating scale ${ }^{16}$ will be used given their use principally as clinical descriptors. Biological samples will include blood, urine and saliva (eg, for cortisol measurements) stored under optimal conditions in the central EPAD Biobank.

\section{EPAD LCS-MINI protocol for participants who maintain a low} likelihood of trial inclusion

During EPAD LCS, it may become clear that some participants maintain a low likelihood of being invited to the PoC trial. This may happen for several reasons, for example, developing health conditions that preclude trial participation or showing no impairment/decline in cognition and $\mathrm{AD}$ biomarkers. Starting from their third visit (1 year after baseline), such participants may have the possibility to continue with a lower-burden protocol, that is, without the yearly MRI and CSF sampling.

\section{Data sources, collection and monitoring}

The only data source for this study will be data collected as part of the EPAD LCS. Electronic data capture will be used as appropriate, for example, for cognitive and imaging data. Central laboratories will be used for all CSF (University of Gothenburg) and genetic (University of Edinburgh) assessments, and central reading of all neuroimaging will be undertaken (University of Edinburgh, VU University Medical Centre Amsterdam). A common pre-analytical procedures schedule for sample collection, storage and shipment will be used at all EPAD LCS sites. The study will be monitored in accordance with the International Conference on Harmonisation (ICH) GCP (ICH Topic E6, 1996).

\section{STATISTICAL ANALYSIS}

\section{Sample size}

To achieve our objective of running a platform trial, we anticipate needing a readiness cohort of several thousand people, that is, the number will be determined by the EPAD PoC trial needs. The EPAD LCS sample size will be maintained through continuous recruitment from PCs and via PrePAD Velocity. Strategies for motivation and engagement as well as improving the research experience for participants will be developed, including, for example, newsletters, websites and telephone contact from the study sites.

\section{Disease modelling}

$\mathrm{AD}$ is a complex condition, and an individual's probability of developing dementia is most likely the result of 
Table 2 European Prevention of Alzheimer's Dementia Longitudinal Cohort Study outcomes and other assessments

$\begin{array}{ll}\text { Primary } & \text { The RBANS Total Scale Index Score based on: } \\ \text { cognitive } & \text { Verbal Episodic Memory: List Learning and Story Memory } \\ \text { outcome } & \text { Visual Episodic Memory: Figure Recall } \\ & \text { Visuospatial/Constructional: Figure Copy and Line Orientation } \\ & \text { Language: Picture Naming } \\ & \text { Attention/Executive Functioning: Semantic Fluency, Digit Span, Coding }\end{array}$

$\begin{array}{ll}\text { Secondary } & \text { Cognitive outcomes } \\ \text { outcomes } & \text { Working memory: Dot Counting (NIH EXAMINER }{ }^{25} \text { 26 } \\ & \text { Choice reaction time and set shifting: Flanker (NIH EXAMINER) } \\ & \text { CSF biomared associate learning: Favourites (University of California, San Francisco }{ }^{27} \text { ) } \\ & \text { Neuroimaging outcomes (MRI) } \\ & \text { Beta-amyloid, total tau, phosphorylated tau } \\ & \text { Hipocampal and whole-brain volume }\end{array}$

Exploratory

outcomes

\section{Cognitive outcomes}

- Allocentric Space: Four Mountains Task (Cambridge University ${ }^{19}$ )

- Navigation in Egocentric Space: Virtual Reality Supermarket Trolley (University College London ${ }^{28}$ ) Other clinical outcomes

- Amsterdam Instrumental Activities of Daily Living Questionnaire 2930

Neuroimaging outcomes

- Multiregion structural MRI analysis

- Functional regional and network measures

\begin{tabular}{|c|c|}
\hline $\begin{array}{l}\text { Other } \\
\text { assessments }\end{array}$ & 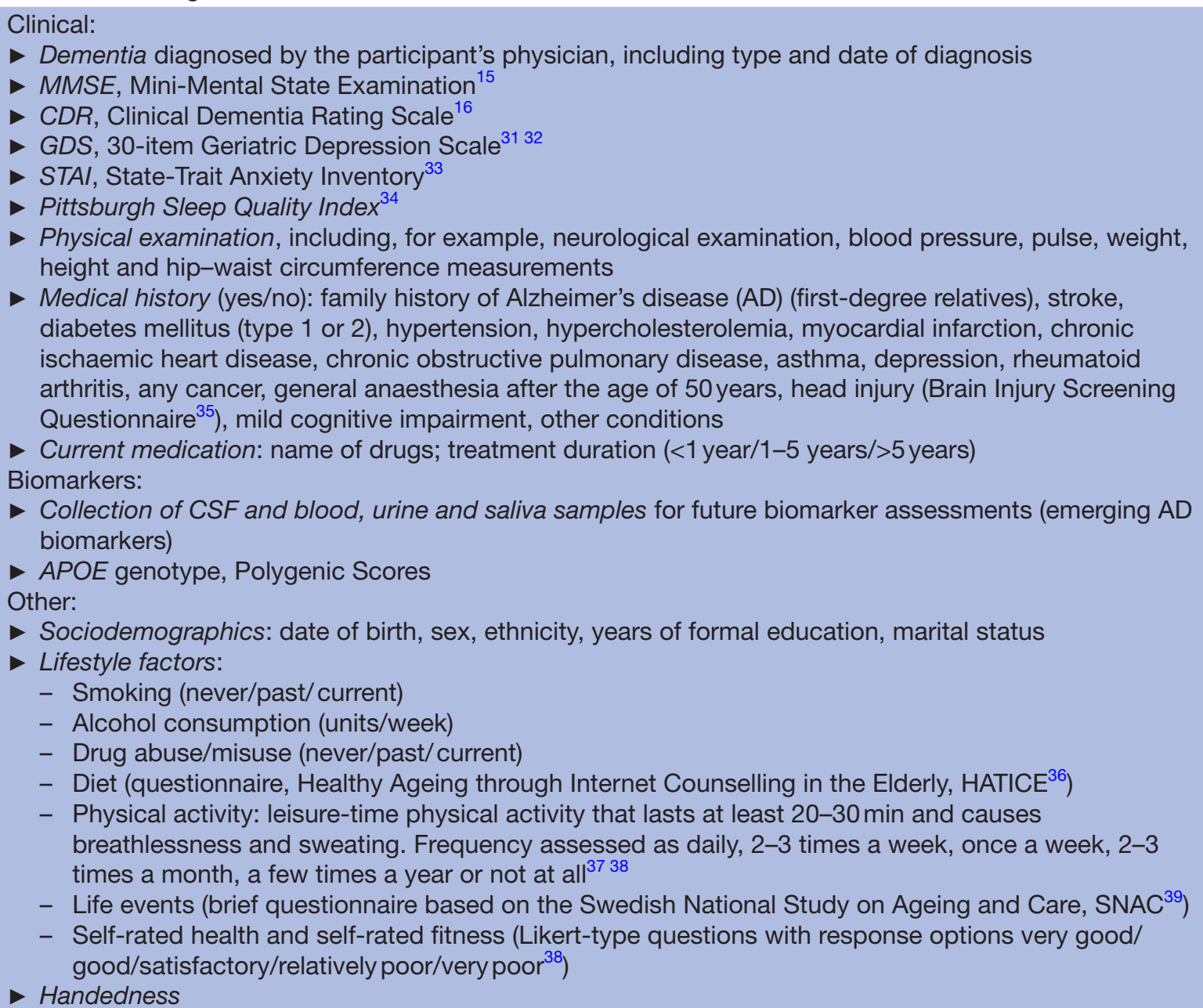 \\
\hline
\end{tabular}

CSF, cerebrospinal fluid; NIH EXAMINER, National Institutes of Health-Executive Abilities: Measures and Instruments for Neurobehavioral Evaluation and Research; RBANS, Repeatable Battery for the Assessment of Neuropsychological Status. 


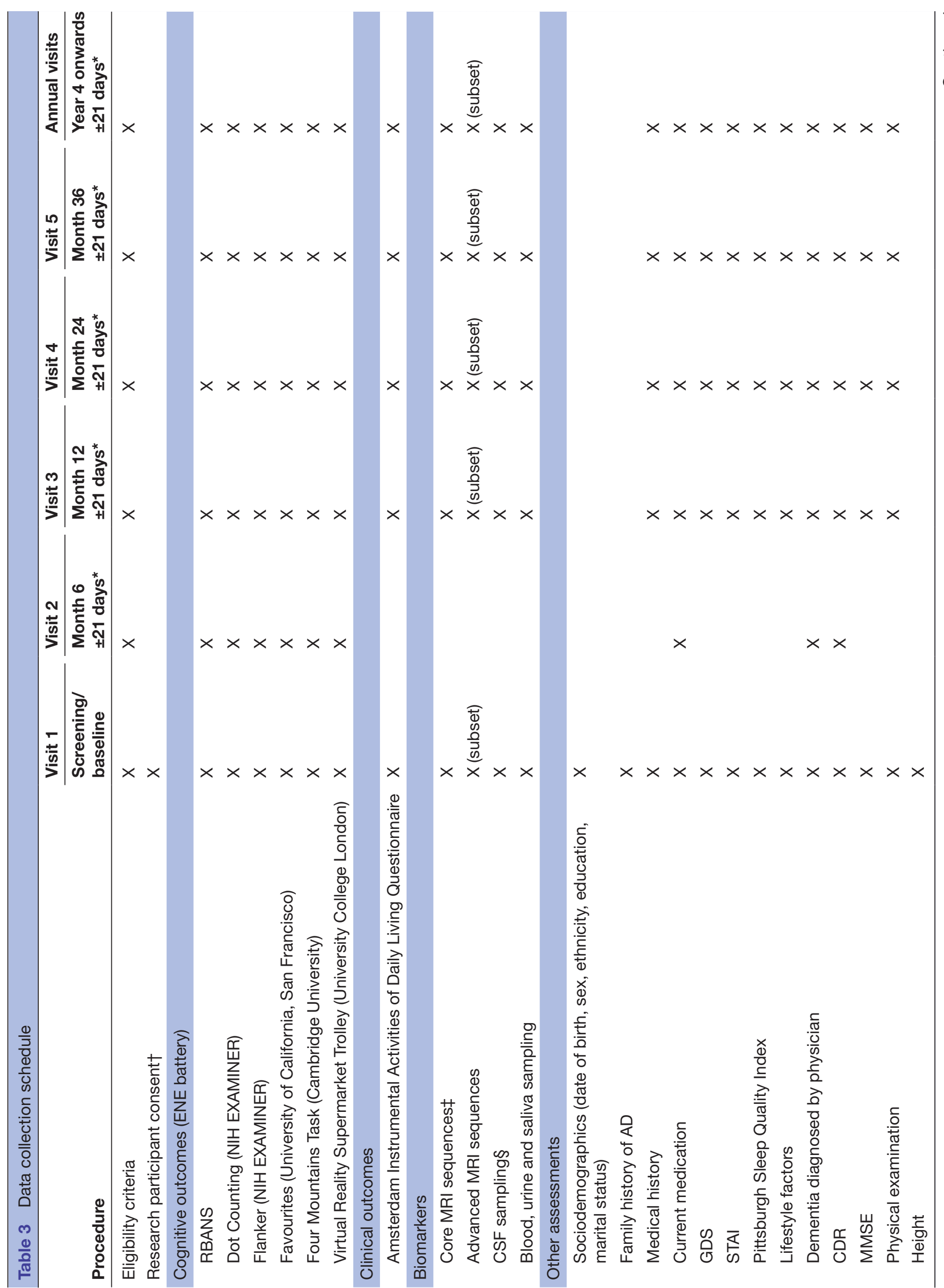




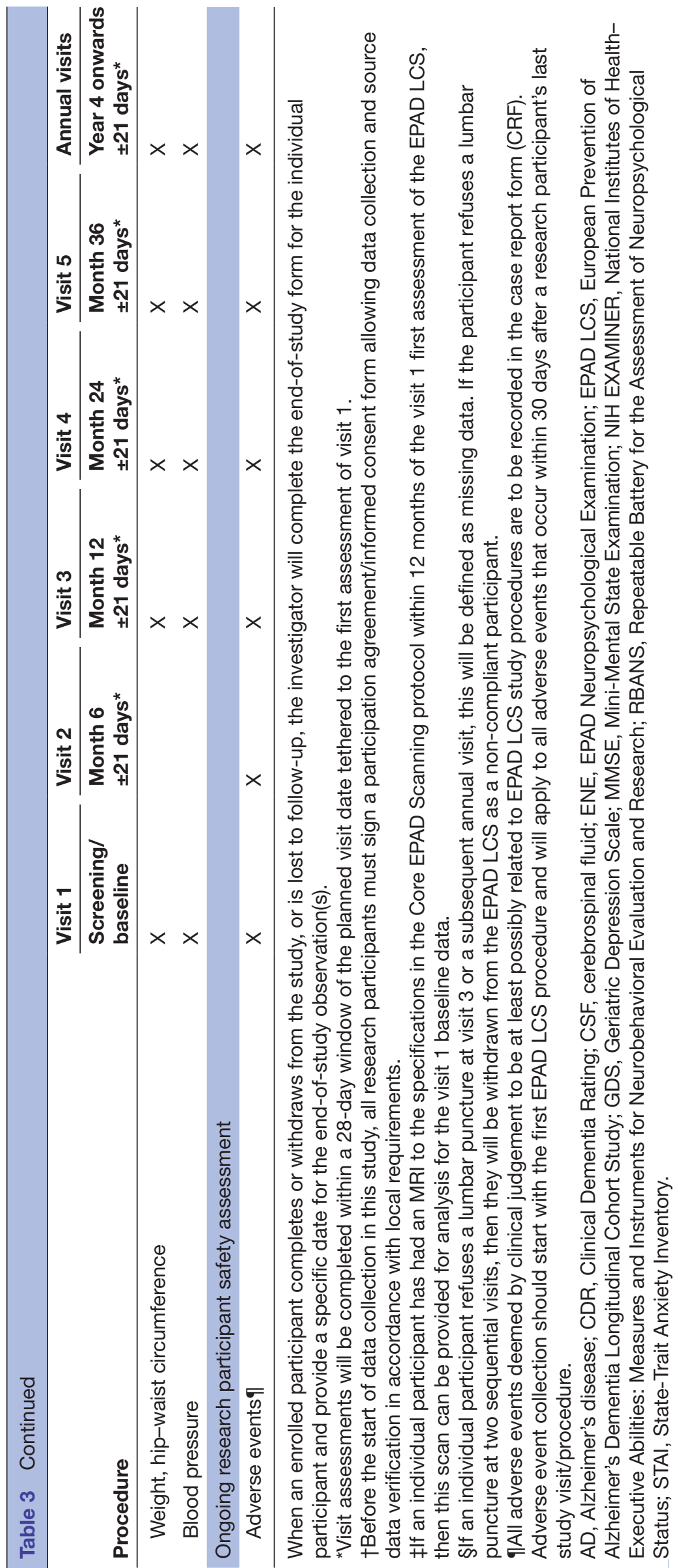


multiple contributing factors. ${ }^{13}$ In EPAD LCS, participants may fall on a continuum of overall probability for subsequent dementia driven by several underlying dimensions: cognition, AD-related biomarkers, traditional risk factors (genetic and environmental) and their longitudinal changes. These dimensions may be continuous in nature. Treating them as such rather than dichotomising or categorising them may result in substantial gains in efficiency and avoidance of information loss when deciding where and why a participant falls in the overall probability continuum. This is especially important as participants with similar overall probability may have different contributions from the various dimensions. Interrogating the underlying dimensions in addition to the overall predicted probability will also facilitate decisions on participant stratification considering the drivers and needs related to compounds to be tested in the EPAD PoC trial.

Longitudinal modelling of cognitive outcomes, biomarkers and risk factors will be used to characterise these dimensions dynamically and relate their trajectories to the probability of $\mathrm{AD}$ dementia development or other meaningful intermediate disease states. Modelling will identify and rank strata of subpopulations of different probability. Each subpopulation will have a cognitive, biomarker and risk factors profile, and this stratification will be used to identify potential interventions, the size of a potential intervention effect and to guide the flow of participants from EPAD LCS into subsequent arms of the PoC trial.

The starting point of the modelling will be mixed-effects models for the cognitive outcomes, biomarkers and risk factors, especially as dementia events are expected to be rare in the first few years of follow-up of participants. Complexity of investigated models will subsequently increase and focus on (multivariate) latent trajectory/class mixed models for the longitudinal outcomes and biomarkers, survival and more general event history models for progression to $\mathrm{AD}$ dementia and joint models linking these longitudinal outcomes and biomarkers to AD dementia. The longitudinal models will initially be developed for each cognitive outcome and biomarker separately and then combined to ultimately maximise the prediction of probability for subsequent dementia.

Analyses of cognitive outcomes will be carried out at both the individual cognitive domain and composite score (RBANS Total Scale Index) levels. Robustness of models developed will be evaluated using cross-validation.

As data accrue in the EPAD LCS, soft data locks and releases will occur after 500, 1000 and 2000 participants (and by intervals of 1000 thereafter) and by stage of follow-up, for example, baseline, 1-year, 2-year and so on, to inform selection algorithms for EPAD LCS; provide updated information for improving selection into the EPAD PoC trial; and provide updated disease models.

\section{ETHICAL ASPECTS}

The study is conducted in full conformance with the principles of the 'World Medical Association Declaration of Helsinki' (52nd WMA General Assembly, Edinburgh, Scotland, October 2000, including the Notes of Clarification as added in 2002, Washington, and 2004, Tokyo, and 2008, Seoul, and 2013, Fortaleza), ICH guidelines for GCP and local legislation of the country in which the research is conducted, whichever affords the greater protection to the individual. EPAD LCS has received ethical approval from numerous institutional review boards across Europe.

EPAD has an Ethics Workgroup examining the complex ethical considerations involved in the project and providing appropriate recommendations. ${ }^{17} 18$

\section{Informed consent}

As the EPAD project is multistaged, staged consent will be used as decision-making model. ${ }^{19}$ Staged consent feeds relevant information-bit by bit, extended over time-to participants and study partners, and asks informed consent at every step when they need to make important decisions. Although informed consent is given for a specific stage of EPAD (eg, consent for LCS does not imply consent for the PoC trial), information about the 'totality of EPAD' will always and explicitly be made available.

\section{Potential disclosure of risk information}

Overall estimated probability for developing AD dementia will not be disclosed to research participants due to insufficient accuracy/robustness of current disease models. However, findings with established clinical relevance and requiring further monitoring and treatment will be disclosed to participants, and appropriate measures will be taken. AD-related CSF biomarkers may be disclosed if progression to $\mathrm{AD}$ dementia is suspected during $\mathrm{EPAD}$ LCS, or where it is considered relevant to an individual's ongoing clinical management, or if a participant is later invited to the PoC trial.

\section{Privacy of personal data}

EPAD LCS will ensure that data on participants are appropriately managed, and participant and study information are treated as confidential. All participant study records are identified by the participant identification number to maintain participants' confidentiality.

\section{RESEARCH PARTICIPANT INVOLVEMENT}

EPAD has established a Research Participants Panel to provide feedback of the experience of research participation, to ensure that participant perspectives are represented in decision-making about the future of the project and to advise local and central EPAD LCS teams. The local panel will consist of 6-10 EPAD LCS participants at each site and will meet at least twice annually. All EPAD LCS participants at a site will be eligible to take part and asked to join the panel for 2 years. A waiting list will be 
maintained of those who are interested if the panel is full. One member of the local panel will also be asked to attend the EPAD General Assembly, to contribute to discussions on study progress, governance and future plans.

\section{DISSEMINATION PLAN}

Findings will be disseminated to several target audiences, including the scientific community, research participants, patient community, general public, industry, regulatory authorities and policy-makers. Types of communication will include scientific publications, conference presentations, press releases, interviews and other media communications (including social media), meetings and so on. Information and regular updates are posted on the EPAD project website (www.ep-ad.org).

Data collected from EPAD LCS will be made available for analysis to help researchers everywhere improve their understanding of the early stages of $\mathrm{AD}$ and facilitate collaborations.

\section{DISCUSSION}

The EPAD project has been established to overcome the major hurdles hampering drug development for the secondary prevention of $\mathrm{AD}$ dementia, by conducting the EPAD LCS in alignment with the Bayesian adaptive designed EPAD PoC trial. This set-up addresses the dual need for (1) developing accurate longitudinal models for $\mathrm{AD}$ covering the entire disease course and (2) developing an adequate infrastructure for facilitating identification of participants and clinical trial recruitment. While several dementia prediction models have already been developed, very few have been validated, and none has been tested in a drug trial. The alignment of a longitudinal cohort study with an adaptive trial design within the same project ${ }^{5}$ is a novel approach that closes the previous gap between dementia prediction and prevention. This design aspect differentiates EPAD LCS from other international networks of observational studies, for example, the World Wide ADNI, ${ }^{20}$ the Integrative Analysis of Longitudinal Studies of Ageing and Dementia (IALSA/Maelstrom), ${ }^{21}$ or Stroke and Cognition consortium (STROKOG).$^{22}$ Other novel solutions for facilitating trial recruitment include, for example, online Brain Health Registers, ${ }^{23}{ }^{24}$ but they require older populations with significant internet literacy, and outcome measures cannot yet be aligned between the online observational cohorts and clinical trials.

EPAD LCS recruitment relies on existing cohorts across Europe. The variety of recruitment sources, that is, from general populations to memory clinics, will ensure that the EPAD LCS probability-spectrum population can cover the entire continuum of probability for $\mathrm{AD}$ dementia development. The yearly EPAD LCS follow-up with comprehensive cognitive, clinical and biomarker assessments will provide a well-phenotyped population, generating high-quality data for updating disease models, for easier identification of individuals suitable for trial inclusion, and for use as trial run-in data and reference for evaluating intervention efficacy. The novel flexible approach to participant selection is designed to balance the disease modelling and adaptive trial design needs. Both EPAD LCS and EPAD PoC trial will be run in an exclusive network of highly selected, expert sites (Trial Delivery Centres) selected on the basis of strictly applied criteria to ensure the highest possible data quality, successful recruitment and adherence to the EPAD principles.

The EPAD project does not operate alone. Together with IMI's EMIF-AD, Amyloid imaging to prevent Alzheimer's disease (AMYPAD), Real world outcomes across the AD spectrum for better care: multi-modal data access platform (ROADMAP), and Organising Knowledge about Neurodegenerative Disease Mechanisms for the Improvement of Drug Development and Therapy (AETIONOMY) projects, it forms a key and major part of the IMI-AD platform. It is also working closely with other, similar initiatives worldwide, including the US-based Global Alzheimer's Platform. The multinational approach and academiaindustry collaborations are essential for advancing knowledge on the entire spectrum of $\mathrm{AD}$ and for finding effective therapies to prevent the onset of dementia.

Acknowledgements The authors thank all members of the EPAD Consortium, local EPAD LCS teams, parent cohort teams and research participants for their contributions to the development, setting up and running of the study.

Collaborators EPAD partners: AC Immune SA (Switzerland); Alzheimer Europe (Luxemburg); Amgen NV (Belgium); Araclon Biotech S.L. (Spain); Aridhia (UK); BarcelonaBeta Brain Research Center (Spain); Berry Consultants LLP (UK); Biogen Idec, Inc. (USA); Boehringer Ingelheim International GmbH (Germany); Cardiff University (UK); Eisai Inc. (USA); Erasmus Universitair Medisch Centrum Rotterdam (Netherlands); Fraunhofer-Gesellschaft zur Förderung der angewandten Forschung e.V. (Germany); Centre Hospitalier Universitaire de Toulouse (France); Hôpital de la Salpêtrière (France); Institut National de la Santé et de la Recherche Médicale (France); IXICO Technologies Ltd (UK); Janssen Pharmaceutica NV (Belgium); Karolinska Institutet (Sweden); Eli Lilly and Company Ltd (UK); H. Lundbeck A/S (Denmark); Medical Research Council (UK); Novartis Pharma AG (Switzerland); Pfizer Limited (UK); IQVIA (UK); Radboud University (Netherlands); F. Hoffmann-La Roche Ltd (Switzerland); Sanofi-Aventis Recherche and Développement (France); Synapse Research Management Partners S.L. (Spain); Takeda Development Centre Europe Ltd (UK); University of Edinburgh (UK); UCB Biopharma SPRL (Belgium); Université de Genève (Switzerland); University of Cambridge (UK); University of Leicester (UK); University of Oxford (UK); Stichting VU-VUmc (Netherlands); Uniklinik Köln (Germany).

Contributors CWR is the chief investigator. AS, MK and JLM are coordinating investigators. BT is the coordinating biostatistician. All authors have contributed to the writing of the study protocol and have approved the final version of this manuscript.

Funding This work has received support from the EU/EFPIA Innovative Medicines Initiative Joint Undertaking EPAD grant agreement 115736. AS has received funding from the Academy of Finland, Stockholm County Council ALF and Alzheimerfonden (Sweden). MK has received funding from Center for Innovative Medicine (CIMED) at Karolinska Institutet (Sweden), Knut and Alice Wallenberg Foundation (Sweden), and Stiftelsen Stockholms sjukhem (Sweden). BT is supported through MRC programme grants MC_UP_1302/3 and MC_UU_00002/2 (UK).

Competing interests None declared.

Patient consent for publication Not required.

Provenance and peer review Not commissioned; externally peer reviewed.

Open access This is an open access article distributed in accordance with the Creative Commons Attribution Non Commercial (CC BY-NC 4.0) license, which permits others to distribute, remix, adapt, build upon this work non-commercially, 
and license their derivative works on different terms, provided the original work is properly cited, appropriate credit is given, any changes made indicated, and the use is non-commercial. See: http://creativecommons.org/licenses/by-nc/4.0/.

\section{REFERENCES}

1. Livingston G, Sommerlad A, Orgeta V, et al. Dementia prevention, intervention, and care. Lancet 2017:67:31363-6.

2. Alzheimer's Disease International World Alzheimer Report 2015. 2017. https://www.alz.co.uk/research/world-report-2015 (Accessed 16 Nov 2017).

3. Winblad B, Amouyel P, Andrieu S, et al. Defeating Alzheimer's disease and other dementias: a priority for European science and society. Lancet Neurol 2016;15:455-532.

4. Jack CR, Knopman DS, Jagust WJ, et al. Update on hypothetical model of Alzheimer's disease biomarkers. Lancet Neurol 2013;12:207-16.

5. Ritchie CW, Molinuevo JL, Truyen L, et al. Development of interventions for the secondary prevention of Alzheimer's dementia: the European Prevention of Alzheimer's Dementia (EPAD) project. Lancet Psychiatry 2016;3:179-86.

6. Dubois $\mathrm{B}$, Feldman $\mathrm{HH}$, Jacova $\mathrm{C}$, et al. Research criteria for the diagnosis of Alzheimer's disease: revising the NINCDS-ADRDA criteria. Lancet Neurol 2007;6:734-46.

7. Dubois $\mathrm{B}$, Feldman $\mathrm{HH}$, Jacova $\mathrm{C}$, et al. Advancing research diagnostic criteria for Alzheimer's disease: the IWG-2 criteria. Lancet Neurol 2014;13:614-29.

8. Albert MS, DeKosky ST, Dickson D, et al. The diagnosis of mild cognitive impairment due to Alzheimer's disease: recommendations from the National Institute on Aging-Alzheimer's Association workgroups on diagnostic guidelines for Alzheimer's disease. Alzheimers Dement 2011;7:270-9.

9. Sperling RA, Aisen PS, Beckett LA, et al. Toward defining the preclinical stages of Alzheimer's disease: recommendations from the National Institute on Aging-Alzheimer's Association workgroups on diagnostic guidelines for Alzheimer's disease. Alzheimers Dement 2011;7:280-92.

10. Dubois $\mathrm{B}$, Hampel $\mathrm{H}$, Feldman $\mathrm{HH}$, et al. Preclinical Alzheimer's disease: definition, natural history, and diagnostic criteria. Alzheimers Dement 2016;12:292-323

11. Hampel H, O'Bryant SE, Castrillo Jl, et al. PRECISION MEDICINEthe golden gate for detection, treatment and prevention of Alzheimer's disease. J Prev Alzheimers Dis 2016;3-

12. Lisa V, Veal CD, Lea terM, et al. European Prevention of Alzheimer's Dementia (EPAD) Registry: recruitment and pre-screening approach for a longitudinal cohort and prevention trials. Alzheimers Dement 2018.

13. Ritchie C, Vermunt L, Solomon A. Ensuring that the EPAD readiness cohort remains 'fit for purpose'. J Prev Alzheimers Dis 2016;3:265.

14. Ritchie K, Ropacki M, Albala B, et al. Recommended cognitive outcomes in preclinical Alzheimer's disease: consensus statement from the European Prevention of Alzheimer's Dementia project. Alzheimers Dement 2017;13:186-95.

15. Folstein MF, Folstein SE, McHugh PR. Mini-mental state. A practical method for grading the cognitive state of patients for the clinician. $J$ Psychiatr Res 1975;12:189-98.

16. Morris JC. The Clinical Dementia Rating (CDR): current version and scoring rules. Neurology 1993;43:2412-4.

17. Milne R, Bunnik E, Tromp K, et al. Ethical issues in the development of readiness cohorts in Alzheimer's disease research. J Prev Alzheimers Dis 2017;4:125-31.

18. Molinuevo JL, Cami J, Carné X, et al. Ethical challenges in preclinical Alzheimer's disease observational studies and trials: results of the Barcelona summit. Alzheimers Dement 2016;12:614-22.
19. Moodley K, Minati L, Contarino V, et al. Diagnostic differentiation of mild cognitive impairment due to Alzheimer's disease using a hippocampus-dependent test of spatial memory. Hippocampus 2015;25:939-51.

20. Hendrix JA, Finger B, Weiner MW, et al. The worldwide Alzheimer's disease neuroimaging initiative: an update. Alzheimers Dement 2015;11:850-9.

21. Integrative Analysis of Longitudinal Studies of Aging (IALSA). http:// www.ialsa.org/ (Accessed 24 Mar 2018).

22. Sachdev PS, Lo JW, Crawford JD, et al. STROKOG (stroke and cognition consortium): an international consortium to examine the epidemiology, diagnosis, and treatment of neurocognitive disorders in relation to cerebrovascular disease. Alzheimers Dement 2017;7:11-23.

23. Zwan MD, Flenniken D, Finley S, et al. The Dutch Brain Health Registry: optimizing recruitment for dementia research. Alzheimer's \& Dementia 2017;13:24.

24. Weiner MW, Veitch DP, Aisen PS, et al. The Alzheimer's Disease Neuroimaging Initiative 3: continued innovation for clinical trial improvement. Alzheimers Dement 2017;13:561-71.

25. Kramer JH, Mungas D, Possin KL, et al. NIH EXAMINER: conceptualization and development of an executive function battery. $J$ Int Neuropsychol Soc 2014;20:11-19.

26. Weintraub S, Dikmen SS, Heaton RK, et al. Cognition assessment using the NIH Toolbox. Neurology 2013;80:S54-64.

27. Hampstead BM, Stringer AY, Stilla RF, et al. Activation and effective connectivity changes following explicit-memory training for facename pairs in patients with mild cognitive impairment: a pilot study. Neurorehabil Neural Repair 2011:25:210-22.

28. Tu S, Wong S, Hodges JR, et al. Lost in spatial translation-a novel tool to objectively assess spatial disorientation in Alzheimer's disease and frontotemporal dementia. Cortex 2015;67:83-94.

29. Sikkes SA, Knol DL, Pijnenburg YA, et al. Validation of the Amsterdam IADL Questionnaire $($, a new tool to measure instrumental activities of daily living in dementia. Neuroepidemiology 2013;41:35-41.

30. Sikkes SA, de Lange-de Klerk ES, Pijnenburg YA, et al. A new informant-based questionnaire for instrumental activities of daily living in dementia. Alzheimers Dement 2012;8:536-43.

31. Yesavage JA, Brink TL, Rose TL, et al. Development and validation of a geriatric depression screening scale: a preliminary report. $J$ Psychiatr Res;17:37-49.

32. Debruyne $H$, Van Buggenhout $\mathrm{M}$, Le Bastard $\mathrm{N}$, et al. Is the geriatric depression scale a reliable screening tool for depressive symptoms in elderly patients with cognitive impairment? Int J Geriatr Psychiatry 2009;24:556-62

33. Julian LJ. Measures of anxiety: State-Trait Anxiety Inventory (STAI), Beck Anxiety Inventory (BAI), and Hospital Anxiety and Depression Scale-Anxiety (HADS-A). Arthritis Care Res 2011;63:S467-72.

34. Buysse DJ, Reynolds CF, Monk TH, et al. The Pittsburgh Sleep Quality Index: a new instrument for psychiatric practice and research. Psychiatry Res 1989;28:193-213.

35. Brain Injury Screening Questionnaire. New York: Research and Training Center on Community Integration, Mount Sinai School of Medicine, 1998.

36. www.hatice.eu (accessed 16 Nov 2017).

37. Rovio S, Kåreholt I, Helkala EL, et al. Leisure-time physical activity at midlife and the risk of dementia and Alzheimer's disease. Lancet Neurol 2005;4:705-11.

38. Kulmala J, Solomon A, Kåreholt I, et al. Association between mid- to late life physical fitness and dementia: evidence from the CAIDE study. J Intern Med 2014;276:296-307.

39. SNAC-K Population Study. http://www.snac-k.se/ (Accessed 16 Nov 2017). 